\title{
State and Training Effects of Mindfulness Meditation on Brain Networks Reflect Neuronal Mechanisms of Its Antidepressant Effect
}

\author{
Chuan-Chih Yang, ${ }^{1,2,3}$ Alfonso Barrós-Loscertales, ${ }^{1}$ Daniel Pinazo, ${ }^{4}$ \\ Noelia Ventura-Campos, ${ }^{1,5}$ Viola Borchardt, ${ }^{2,3}$ Juan-Carlos Bustamante, ${ }^{6}$ \\ Aina Rodríguez-Pujadas, ${ }^{1}$ Paola Fuentes-Claramonte, ${ }^{1}$ Raúl Balaguer, ${ }^{4}$ \\ César Ávila, ${ }^{1}$ and Martin Walter ${ }^{2,3,7,8,9}$ \\ ${ }^{1}$ Departamento de Psicología Básica, Clínica y Psicobiología, Universitat Jaume I, 12071 Castellón, Spain \\ ${ }^{2}$ Clinical Affective Neuroimaging Laboratory, 39120 Magdeburg, Germany \\ ${ }^{3}$ Department of Behavioral Neurology, Leibniz Institute for Neurobiology, 39118 Magdeburg, Germany \\ ${ }^{4}$ Departamento de Psicología Evolutiva, Educativa, Social y Metodología, Universitat Jaume I, 12071 Castellón, Spain \\ ${ }^{5}$ Departamento de Didáctica de la Matemática, Facultad de Magisterio de la Universidad de Valencia, 46022 Valencia, Spain \\ ${ }^{6}$ Departamento de Psicologia y Sociologia, Universidad de Zaragoza, 50009 Zaragoza, Spain \\ ${ }^{7}$ Department of Psychiatry and Psychotherapy, Otto-von-Guericke University, 39120 Magdeburg, Germany \\ ${ }^{8}$ Center for Behavioral Brain Sciences (CBBS), 39120 Magdeburg, Germany \\ ${ }^{9}$ Department of Psychiatry and Psychotherapy, Eberhard Karls University, 72076 Tuebingen, Germany
}

Correspondence should be addressed to Alfonso Barrós-Loscertales; barros@uji.es

Received 17 August 2015; Revised 10 January 2016; Accepted 11 January 2016

Academic Editor: Bruno Poucet

Copyright (C) 2016 Chuan-Chih Yang et al. This is an open access article distributed under the Creative Commons Attribution License, which permits unrestricted use, distribution, and reproduction in any medium, provided the original work is properly cited.

\begin{abstract}
The topic of investigating how mindfulness meditation training can have antidepressant effects via plastic changes in both resting state and meditation state brain activity is important in the rapidly emerging field of neuroplasticity. In the present study, we used a longitudinal design investigating resting state fMRI both before and after 40 days of meditation training in 13 novices. After training, we compared differences in network connectivity between rest and meditation using common resting state functional connectivity methods. Interregional methods were paired with local measures such as Regional Homogeneity. As expected, significant differences in functional connectivity both between states (rest versus meditation) and between time points (before versus after training) were observed. During meditation, the internal consistency in the precuneus and the temporoparietal junction increased, while the internal consistency of frontal brain regions decreased. A follow-up analysis of regional connectivity of the dorsal anterior cingulate cortex further revealed reduced connectivity with anterior insula during meditation. After meditation training, reduced resting state functional connectivity between the pregenual anterior cingulate and dorsal medical prefrontal cortex was observed. Most importantly, significantly reduced depression/anxiety scores were observed after training. Hence, these findings suggest that mindfulness meditation might be of therapeutic use by inducing plasticity related network changes altering the neuronal basis of affective disorders such as depression.
\end{abstract}

\section{Introduction}

Mindfulness meditation has been shown to be therapeutic in emotion regulation [1-5]. Mindfulness practice, inherited from the ancient Buddhist tradition, involves observing thoughts nonjudgmentally in the present moment.
Mindfulness-based meditation (MBM) or mindfulnessbased intervention (MBI) is increasingly being employed in western psychology to alleviate a variety of mental and physical conditions including obsessive compulsive disorder and anxiety and in the prevention of relapse in depression and drug addiction [6-10]. Despite the fact that recent 
neuroimaging studies indicate the several positive aspects of mindfulness practice, its neuronal mechanisms are still poorly understood.

A main theme in meditation research is the question if the observed effects are dependent on practice/expertise or on personal characteristics. Usually, studies on meditation compare expert practitioners with novice practitioners in crosssectional studies [11-13]. Functional studies have employed fMRI block designs to investigate the blood-oxygen level dependent (BOLD) signal changes during meditation [11, $12,14]$, as well as exploring resting state fMRI (rs-fMRI) differences between experienced meditators and novices [11, $13,15]$. Crucially, longitudinal studies of the impact of shortterm meditation practice on the same novice group have the potential to reflect effects of meditation independently of individual differences, for example, personal interest in meditation or previous meditation experience. Hölzel et al. [4] observed changes in gray matter density in a longitudinal study with 8 weeks of a mindfulness-based stress reduction (MBSR) programme.

Resting state functional connectivities (RSFC) methods provide one approach for exploring how mindfulness meditation alters neural plasticity among brain regions. The interregional dynamics of RSFC specifically afford the advantages of being task independent, rendering reliable estimates of neural circuit functionality corresponding to structural topography $[16,17]$.

To investigate the impact of mindfulness practice on the neuronal level and its antidepressant effects, alteration in the activation pattern of the Default Mode Network (DMN) is of particular interest and the DMN is thus selected as the target brain network [13]. We know that the DMN involves several brain regions active when the brain is not actively engaged in a cognitively demanding task but rather is in a relaxed state [18]. Consistently, the DMN includes areas at the medial posterior cortex, specifically the posterior cingulate cortex (PCC; areas 23/31), the precuneus, and the medial frontal cortex (MFC, including areas 24/10-m/32), as well as bilateral inferior parietal and posterior temporal areas around the temporoparietal junction area (TPJ) [19].

Recent findings suggested that the DMN has altered resting state functional connectivity between networks when depressed patients were compared to healthy participants [20]. Among these DMN regions, dmPFC was shown to be involved in self-inspection [21] and emotion regulation [22] and demonstrated increased activity in depression [23]. Thus, major depressive disorder (MDD) patients show more neural functional connectivity between the posterior cingulate cortex and the subgenual-cingulate cortex during rest periods compared to healthy individuals [24].

Several regions of anterior cingulate are of importance in depression-related disorders. Pregenual anterior cingulate cortex (pgACC) has been shown to be hypoactivated in MDD [25]. The dorsal anterior cingulate cortex (dACC) is associated with the involvement of cognitive control over attentional resources [26, 27], and it is strongly impaired in MDD. van Tol et al. [28] showed that dACC and pgACC are also structurally affected in MDD, while pgACC was furthermore found to be molecularly affected by altered glutamate concentrations. In contrast to the dACC, the pgACC belongs to an affective subdivision of the ACC [26] and was shown to mediate the increased internal focus present in the ruminative thinking style of depressed patients. Furthermore, the pgACC is specifically impaired in highly anhedonic patients [29].

The neurobiological model of depression [30] states that impaired cognitive control, mediated by regions in the prefrontal cortex (PFC), cooccurs with hyperactivation of the amygdala, which facilitates encoding and retrieval of emotional stimuli via modulation of hippocampal activity. Activity in the medial prefrontal cortex (MPFC), which is associated with internal representations of the self, is consequently increased as well. The suppressed regulatory influence of PFC regions facilitates an undesired recall of negative (mood-congruent) events. The DMN has been shown to support internally oriented and self-referential thoughts and MDD has been associated with both hyperconnectivity within the DMN and hyperconnectivity between frontoparietal control systems and regions of the default network [31]. Furthermore, MDD has been characterized by hypoconnectivity within the frontoparietal network, a set of regions involved in cognitive control of attention and emotion regulation, and hypoconnectivity between frontal systems and parietal regions of the dorsal attention network involved in attending to the external environment [31]. These networks modulate affective and cognitive processes disturbed in depression.

In the cases described here, impairment refers to increase or decrease of an existing functional connection. Alterations reflect changes in operational properties of interregional connections and their local resting state activity. As much as MDD is characterized by hyperactivity in, for example, ventral and subcortical regions, this was discussed to be directly related to hypoactivity and resulting disinhibition from dorsal cortical regions [30]. Likewise, within-network connectivity can be altered in opposite direction compared to between-network connectivity (in terms of functional connectivity) as soon as a hub region is concerned, which connects both to its own functional module and towards regions outside its own functional subnetwork.

Recent neuroimaging publications benefit from the utilization of multiple imaging methods. Resting state fMRI widely used seed-based correlation analysis between regions of interest (ROIs) by investigating interregional functional connectivity to reveal highly consistent patterns of functional connectivity across regions [32]. In this study we selected two ROIs in the ACC, pgACC, and dACC, due to their role in emotion regulation and as portions of the DMN. Earlier studies showed that the pgACC was associated with internal monitoring [33, 34] and Mood States [35]. Likewise, the dACC has been shown to relate to focused attention [26, 27] and recent study from Dickenson and colleagues had found that the dACC was recruited during mindfulness meditation as compared to the mind wandering for the novice practitioner [36]. Both pgACC and dACC are therefore target regions for specific symptoms in depression related to mindfulness and emotional/interoceptive awareness, and we focused our analysis on these two regions with supposedly differential, 
if not contrary, effects, as previously reported in patient studies and suggested by their involvement in potentially antagonistic networks. The method of seed-based functional connectivity was used to test their resting state behavior both before and after training. Furthermore, independent component analysis (ICA), which neither relies on a priori model of brain activity, parcellation into ROIs, nor choice of seed regions but has been widely used to detect resting state networks [37], was chosen as a complementary, datadriven approach. We hypothesized that some regions within DMN would be relevant to emotional regulation and ICA was used for functional parcellation of the DMN. In addition to seed-based functional connectivity method and ICA, which reflect the interregional neuronal properties between distant brain regions, ReHo was used to map the level of regional activity synchronization across the whole brain [38]. Regional Homogeneity (ReHo) measures the similarity of the time series of a given voxel to those of neighbouring voxels [39], which reflects the temporal homogeneity of localized neural dynamics [40-42].

Therefore, our aims were to analyze both the interregional and local features of fMRI signal during resting state (RS) in a longitudinal study of RS activation, as well as comparing RS activation to meditation state (MS) activation. We hypothesized that the training of mindfulness meditation will lead to changes in the following: (1) the interregional functional connectivity of our a priori seed ROIs-pgACC and AACC (by means of seed-based FC); (2) the internal consistency of the DMN (by means of ICA); and (3) the regional synchronization of fMRI time series (by means of $\mathrm{ReHo}$.

\section{Materials and Methods}

2.1. Subjects. Thirteen university students (Spanish native speakers) were recruited by university advertisement to participate in 40 days of mindfulness meditation course. Sample demographic characteristics are detailed in Table 1. All study protocols were approved by the Institutional Review Board of the Universitat Jaume I of Castellón and informed consent was obtained from all subjects. Participants were screened for psychiatric or neurological conditions prior to enrollment in the meditation course. No previous meditation experience was reported by the subjects.

2.2. Mindfulness Meditation Training. The mindfulness training programmes consisted of 8-week courses, with daily practice at home in sessions of around 45 minutes [43]. The mindfulness-based stress reduction (MBSR) programme [44] as well as acceptance and commitment therapy [45] was used to design the meditation-mindfulness training programme based on self-observation training. The selfobservation training programme consisted of eight 1.5-hour sessions over a period of 8 weeks. The first hour of the sessions was devoted to simple physical and breathing exercises during which the participants were instructed to perform Vipassana meditation exercises, by focusing their attention on thoughts that came to their mind without dwelling on any of them. After the exercises, participants meditated in silence,
TABLE 1: Characteristics of the subject sample. Wherever relevant, group mean ( \pm standard deviation) is given.

\begin{tabular}{lc}
\hline Number of participants & 13 \\
Ratio of males and females & $3 / 10$ \\
Age & $24.53( \pm 5.89)$ \\
Ratio of handedness & right $=13$, left $=0$ \\
\hline $\begin{array}{l}\text { Minutes of meditation practice per day } \\
\text { Total number of meditation days until }\end{array}$ & $10.67( \pm 1.66)$ \\
$\begin{array}{l}\text { scanning } \\
\text { Total number of minutes of meditation } \\
\text { practice until scanning }\end{array}$ & $39.23( \pm 3.63)$ \\
\hline
\end{tabular}

continuing to observe their thoughts without censoring them. The time devoted to meditation without the physical exercises gradually increased over the course. The final half hour was used to talk about the experience and explain the characteristics of meditation and mindfulness in which videos and fables reflecting the most significant aspects of the meditation and self-observation experience were used. Participants were also encouraged to do the meditation exercises at home; they were given a meditation diary in which to record their daily experiences, which was handed in to the course instructor. A quantification of the participants' amount of meditation practice at the end of the course is detailed in Table 1.

2.3. Self-Report Measures. Three self-assessment questionnaires were administrated to participants both before and after the training period. We applied the Profile of Mood States (POMS) throughout the study in its abbreviated, Spanish version [46] of the original POMS [47]. It is a 44-item inventory, which measures current mood state by rating statements on a Likert scale (0 to 4). It consists of six subscales: anger, fatigue, tension, depression, vigor, and friendliness. To evaluate changes in depressive symptoms of our sample, we used the Center for Epidemiologic Studies Depression Scale (CES-D) which is a well-validated, 20-item inventory [48]. Subjects were asked to rate statements based on the previous week on a Likert scale (0-3) and scores range from 0 to 60 , whereat higher scores indicate higher levels of depression. The State-Trait Anxiety Inventory [49] is a 40-item scale designed to measure the state and trait anxiety based on a Likert scale (1-4), which was also applied. Scores range from 20 to 80 whereat higher scores relate to higher levels of anxiety.

2.4. Data Acquisition. In this longitudinal study participants were scanned twice. Before the training of mindfulness meditation, subjects underwent a single resting state (RS) scan (time point 1, TP1). Later on, at day 40 (time point 2, TP2), subjects underwent two scans, RS scan and a scan during which they practiced meditation (MS). During the RS, subjects were instructed to close their eyes, be at the normal relaxing condition without engaging in any specific task or mental activity, and not fall into meditation. During meditation scan, subjects were instructed to close their eyes, openly monitor the surrounding environment by accepting all sensations rise and fall nonjudgmentally, and specifically to be aware of the present moment as they were trained. At 
time point 2 (TP2), RS scan was measured for 9 minutes followed by continuous MS lasting for 12 minutes. Long time acquisition for both conditions was set to obtain sufficient power for the functional connectivity analyses given the relatively small sample size [37].

2.5. MR Sequence Parameters. MR measurements were performed on 1.5 T Siemens AVANTO scanner (Siemens Erlangen, Germany). A structural image was acquired from each subject with a magnetization-prepared rapid gradient-echo $(\mathrm{MP}-\mathrm{RAGE})$ sequence $(\mathrm{TR}=2200 \mathrm{~ms}, \mathrm{TE}=3.79 \mathrm{~ms}$, flip angle $(\mathrm{FA})=15^{\circ}, 160$ slices, matrix size $=256 \times 256$, fieldof-view $($ FOV $)=256 \mathrm{~mm} \times 256 \mathrm{~mm}$, and slice thickness $=$ $1 \mathrm{~mm}$ ). For both RS and MS, a standard EPI sequence was used $\left(\mathrm{TR}=2300 \mathrm{~ms}, \mathrm{TE}=55 \mathrm{~ms}, \mathrm{FA}=90^{\circ}, \mathrm{FOV}=224 \mathrm{~mm} \times\right.$ $224 \mathrm{~mm}$, matrix size $=64 \times 64$, and slice thickness $=4 \mathrm{~mm}$,) with 25 axial slices for whole brain coverage. Finally, an extra gradient field mapping sequence (gre_field_mapping) was acquired followed by each EPI sequence (TR $=487 \mathrm{~ms}$, $\mathrm{TE} 1=8 \mathrm{~ms}, \mathrm{TE} 2=12.76 \mathrm{~ms}, \mathrm{FA}=65^{\circ}, \mathrm{FOV}=224 \mathrm{~mm} \times$ $224 \mathrm{~mm}$, matrix size $=64 \times 64$, and slice thickness $=4 \mathrm{~mm}$ ) with 25 slices with the same coverage used in EPI sequence.

2.6. Data Preprocessing. B0 inhomogeneity correction was performed to reduce static field inhomogeneity using an EpiUnwarping tool based on FSL (http://surfer.nmr.mgh .harvard.edu/fswiki/epidewarp.fsl) [50]. Preprocessing was performed using Statistical Parametric Mapping (SPM8, http://www.fil.ion.ucl.ac.uk/spm/) and Data Processing Assistant for resting state fMRI (DPARSF v 2.3 [51]). Functional images were slice-time corrected. Motion correction was performed by using a least squares approach and a sixparameter (rigid body) linear transformation. Spatial normalization to MNI space was carried out by using unified segmentation of T1-weighted acquired images, and the extracted normalization parameters from segmentation were applied to normalize the functional volumes for each participant (normalized images were then resampled to 3-mm isotropic cubic voxels). Finally, functional volumes were smoothed by applying a $4 \mathrm{~mm}$ FWHM Gaussian kernel. Smoothed volumes were used for ICA and seed-based FC. ReHo analysis was performed on nonsmoothed functional volumes and images were smoothed after the analysis, because smoothing prior to ReHo calculation would increase regional similarity [51].

We conducted additional preprocessing for seed-based FC and ReHo analyses using the DPARSF tool through the following steps: (i) removing a linear trend in the time series and (ii) temporal band-pass filtering $(0.01-0.08 \mathrm{~Hz})$ to reduce the effect of low frequency drift and high-frequency noise $[52,53]$. For the seed-based FC analysis, several sources of spurious variance were removed from the data through linear regression: six parameters from rigid body correction of head motion, white matter signal, cerebrospinal fluid signal, and the global mean signal [54].

For MS, the first and the last 40 volumes of the functional images were discarded (total 313 volumes were acquired before removing the 80 volumes). The reason for removing the first 40 volumes was to allow the participants to get used

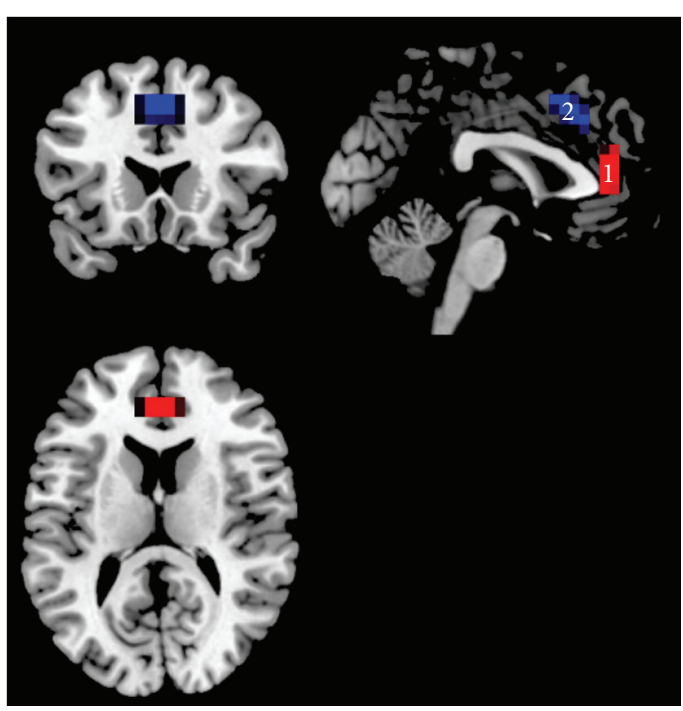

FIgURE 1: Seed ROIs used for RSFC analysis. ROI1: pregenual ACC (pgACC) as marked in red color. ROI2: dorsal ACC (dACC) as marked in blue color overlaid on the T1 anatomical MNI space.

to the scanning environment and ensure a reasonable establishment of the meditation state. The reason for removing the last 40 volumes was aiming at avoiding distraction effects at the end of the meditation, yielding a duration similar to the RS. The central 233 ( $8 \mathrm{~min} 33 \mathrm{sec}$ ) functional volumes of the times series were sufficient for estimation of independent components (ICs) [37].

2.7. Seed-Based Functional Connectivity. In addition to a data-driven approach, we performed a hypothesis-driven seed-based FC analysis. Two independent seed ROIs placed in the pregenual anterior cingulate cortex (pgACC) and the dorsal anterior cingulated cortex (dACC) were chosen based on previous reports on their involvement in meditation and affective disorders such as depression [55].

To examine FC in the pgACC and the dACC in a whole brain voxel-wise analysis, these two seed regions were defined by the Montreal Neurological Institute (MNI) coordinates $(x, y, z): 0,41$, and 9 (pgACC) and 0, 27, and 30 (dACC). Both ROIs had a radius of $10 \mathrm{~mm}$ resulting in a volume of approximately $4 \mathrm{~mL}$, maximizing the gray matter contribution as described previously [56], as shown in Figure 1. The averaged time course from each seed region was obtained for each subject at both time points and for both conditions (RS, MS) separately. An individual FC map for each seed was generated by calculating the voxel-wise correlation coefficients in the whole brain, which were then converted into $z$-maps by Fisher's $Z$ transformation to enhance normality. The $z$-maps of the dACC and pgACC of each individual were entered into second-level paired $t$-test.

2.8. Group Independent Component Analysis (Group ICA). A group independent component analysis was performed using GIFT (v 2.0e, http://mialab.mrn.org/software/gift/) [57] to investigate the following: (a) the training-related RSFC changes and (b) the FC difference between RS and MS. 
These analyses were performed based on FC analysis from spontaneous BOLD activity from the average fMRI time course of the entire brain.

In the analysis of training-related RSFC changes over time, the longitudinal resting state data were grouped together when conducting group ICA following a previous report [58]. In the analysis of FC differences between RS and MS experimental conditions, a single ICA was performed at the group level for RS and MS conditions separately, in agreement with previous studies $[59,60]$. The data of each condition was restricted to twenty-four independent components (ICs) using the MDL criteria and extracted by ICA decomposition using the Infomax algorithm [61]. To determine the reliability of the ICA algorithm, multiple runs of ICA were performed using ICASSO. Each IC consists of a spatial map and an associated time course. The ICs which resembled the DMN most were firstly spatially sorted by using the DMN template built-in in GIFT [57] and then further identified by visual inspection by two different raters for RS and MS separately. Subsequently, individual subject maps were back reconstructed to obtain single-subject results. Finally, the resulting individual IC maps were converted into voxel-wise $z$-score maps representing the degree to which each voxel belongs to the overall ICA component map.

Individual subject $z$-maps were entered into SPM random-effect analyses. The RSFC analysis involved a longitudinal analysis of changes in temporal correlations in connectivity fluctuations before and after training. To compare RS and MS conditions, one paired $t$-test was performed. In the IC decomposition, only DMN was extracted to be compared between time points and conditions. We computed the DMN from the average fMRI time course from the entire network as described before. Furthermore, in the analysis between RS and MS, the DMN comparison was not restricted to a mask of the conjunction between both conditions, since it would involve the restriction of any other regions that may be identified as the DMN in the MS as different from the DMN in the RS, but to the addition of the masks for the DMN identified under RS and MS.

Additionally, exploratory mask restricted analysis was performed but not reported because its contribution was similar to the unrestricted comparison in the target regions of the DMN, although covering differences in other out regions.

2.9. Regional Homogeneity (ReHo). ReHo images of the whole brain for both MS and RS were generated using DPARSF. Voxel-by-voxel Kendall's coefficient of concordance (KCC, [39]) of the time series of a given cluster of 27 neighbouring voxels was calculated [62]. To reduce the global effects of individual variability across participants, the ReHo of each voxel was scaled by the mean value of whole brain ReHo for each participant [20].

A paired $t$-test was performed to identify the effects of meditation on ReHo during resting state activity between TP1 and TP2. A second paired $t$-test was performed to identify the differences in ReHo between the resting states (RS) and meditation state (MS) at TP2. The resulting $t$-value maps of each contrast of interest was displayed after applying a statistical height threshold of $p<0.001$ for each voxel and
TABle 2: Profile of Mood States (POMS), Center for Epidemiologic Studies Depression Scale (CES-D), and State-Trait Anxiety Inventory (STAI) scores before and after meditation training. Group means and standard deviations are listed. Significant group differences are listed as follows: $* * *$ : significant at $p<0.001, * *$ : significant at $p<0.01$, and $*$ : significant at $p<0.05$. $*$ : before training, 6 out of 13 subjects surpassed the cutoff for depression. (+): positive mood state factor, $(-)$ : negative mood state factor.

\begin{tabular}{lcc}
\hline Questionnaire & Before training & After training \\
\hline POMS & & \\
$\quad$ Anger $(-)$ & $2.84( \pm 3.78)$ & $3.30( \pm 6.07)$ \\
Fatigue $(-)$ & $4.84( \pm 2.67)$ & $3.69( \pm 4.57)$ \\
Vigor $(+)$ & $11.92( \pm 5.31)$ & $12.30( \pm 5.36)$ \\
Friendliness $(+)$ & $18.92( \pm 2.98)$ & $19.46( \pm 4.27)$ \\
Tension $(-)$ & $8.00( \pm 3.36)$ & $5.38( \pm 5.18)^{*}$ \\
$\quad$ Depression $(-)$ & $3.76( \pm 3.94)$ & $4.53( \pm 8.04)$ \\
\hline CES-D & $16.23( \pm 9.54)^{*}$ & $9( \pm 6.20)^{* * *}$ \\
\hline STAI, state & $17.84( \pm 4.75)$ & $14.38( \pm 10.16)$ \\
STAI, trait & $21.30( \pm 8.6)$ & $16.84( \pm 9.56)^{* *}$ \\
\hline
\end{tabular}

a corrected cluster threshold at $p<0.05$, as determined by a Monte Carlo simulation (see AlphaSim in AFNI http://afni .nimh.nih.gov/pub/dist/doc/manual/AlphaSim.pdf).

\section{Results}

3.1. Behavioral Self-Report Measures. A paired $t$-test analysis of the total scores of CES-D indicated a significant reduction of depression scores after meditation training $(t(12)=4.43$; $p<0.001)$. Before meditation training, the mean CES-D score of the sample was $16.23 \pm 9.54$, which slightly surpassed the cutoff score for depression (a CES-D above 16 indicates depression; see [63]). After training, the mean CES-D score was reduced to $9 \pm 6.20$.

Pre-post comparisons of the STAI measures revealed a significant reduction in trait anxiety $(t(12)=2.76 ; p<0.01)$, but not in state anxiety scores. Neither the sum score of the POMS nor its sub-scores showed a significant change (all $p<0.05$, controlled for multiple comparisons). However, the tension subscore did show a change $(t(12)=1.883, p<0.05$, uncorrected); see Table 2.

As an exploratory step, the changes in CES-D scores were correlated with the changes in connectivity of the FC components, but the results were not significant for dACC $(r$ $=.122, p>0.1)$ and $\operatorname{pgACC}(r=-.064, p>0.1)$.

\subsection{Seed-Based Functional Connectivity}

3.2.1. Comparison of FC Differences between RS and MS. For the contrast between resting and meditation states (RS $>M S$ ), the pgACC showed a reduced connectivity with the bilateral inferior parietal gyri but an increased connectivity with the MPFC, the left superior temporal gyrus (STG), and the right TPJ during MS as shown in Figure 2(a) and Table $3(p<0.05$, FWE-corrected). 
TABLE 3: Regions showing functional differences between conditions (rest versus meditation).

\begin{tabular}{|c|c|c|c|c|c|c|c|c|c|c|}
\hline \multirow{2}{*}{ Analysis } & \multicolumn{5}{|c|}{ Rest $>$ meditation } & \multicolumn{5}{|c|}{ Meditation $>$ rest } \\
\hline & Region & Side & $K$ & MNI coordinates & $z$-score & Region & Side & $K$ & MNI coordinates & $z$-score \\
\hline \multirow[t]{6}{*}{ ICA } & \multicolumn{10}{|c|}{$\left(p<0.001, \mathrm{FWE}^{\mathrm{a}} \text {-corrected cluster threshold at } p<0.05\right)^{\mathrm{b}}$} \\
\hline & dACC & $\mathrm{R}$ & 202 & 92136 & 5.70 & Precuneus & $\mathrm{R}$ & 178 & $3-6627$ & 4.94 \\
\hline & sgACC & $\mathrm{R}$ & 349 & 9270 & 5.60 & TPJ/IPL & $\mathrm{L}$ & 157 & $-51-5727$ & 4.31 \\
\hline & AI & $\mathrm{L}$ & 336 & -3990 & 5.02 & & & & & \\
\hline & $\mathrm{AI}$ & $\mathrm{R}$ & 37 & 45180 & 4.34 & & & & & \\
\hline & IFG/BA9 & $\mathrm{R}$ & 21 & 601827 & 4.26 & & & & & \\
\hline Seed-based FC & \multicolumn{10}{|c|}{$(p<0.001, \text { FWE-corrected cluster threshold at } p<0.05)^{\mathrm{b}}$} \\
\hline \multirow[t]{3}{*}{ (ROI: pgACC) } & IPL & $\mathrm{L}$ & 42 & $-45-3936$ & 4.31 & TPJ & $\mathrm{R}$ & 58 & $57-6636$ & 4.51 \\
\hline & IPL & $\mathrm{R}$ & 45 & $39-5151$ & 4.17 & MPFC & $\mathrm{R}$ & 36 & 9519 & 4.49 \\
\hline & & & & & & STG & $\mathrm{L}$ & 23 & $-42-6018$ & 3.86 \\
\hline (ROI: dACC) & $\mathrm{AI}$ & $\mathrm{L}$ & 20 & -36183 & 4.2 & n.s. & & & & \\
\hline \multirow[t]{4}{*}{ ReHo } & \multicolumn{10}{|c|}{$(p<0.001 \text {, FWE-corrected cluster threshold at } p<0.05)^{\mathrm{b}}$} \\
\hline & n.s. & & & & & dACC & $\mathrm{R}$ & 125 & 03615 & 5.32 \\
\hline & & & & & & MPFC & $\mathrm{R}$ & 125 & 64812 & 4.24 \\
\hline & & & & & & Putamen & $\mathrm{L}$ & 77 & -2163 & 4.40 \\
\hline
\end{tabular}

Notes: AI, anterior insula; dACC, dorsal anterior cingulate cortex; IPL, inferior parietal lobe; K, cluster size; MPFC, medial prefrontal cortex; pgACC, pregenual anterior cingulate cortex; sgACC, subgenual anterior cingulate cortex; STG, superior temporal gyrus; TPJ, temporoparietal junction; and BA: brodmann area. ${ }^{a}$ Family-wise error.

${ }^{\mathrm{b}}$ A combined threshold of $p<0.001$ and a minimum cluster size determined by AlphaSim ${ }^{\mathrm{c}}$ algorithm in AFNI, resulting in FWE-corrected threshold of $p<$ 0.05. An estimate of the spatial correlation across voxels was modeled using the program $3 \mathrm{dFWHM}^{\mathrm{d}}$ in AFNI.

${ }^{c}$ AlphaSim: http://afni.nimh.nih.gov/pub/dist/doc/manual/AlphaSim.pdf.

d 3dFWHM: http://afni.nimh.nih.gov/pub/dist/doc/program_help/3dFWHM.html.

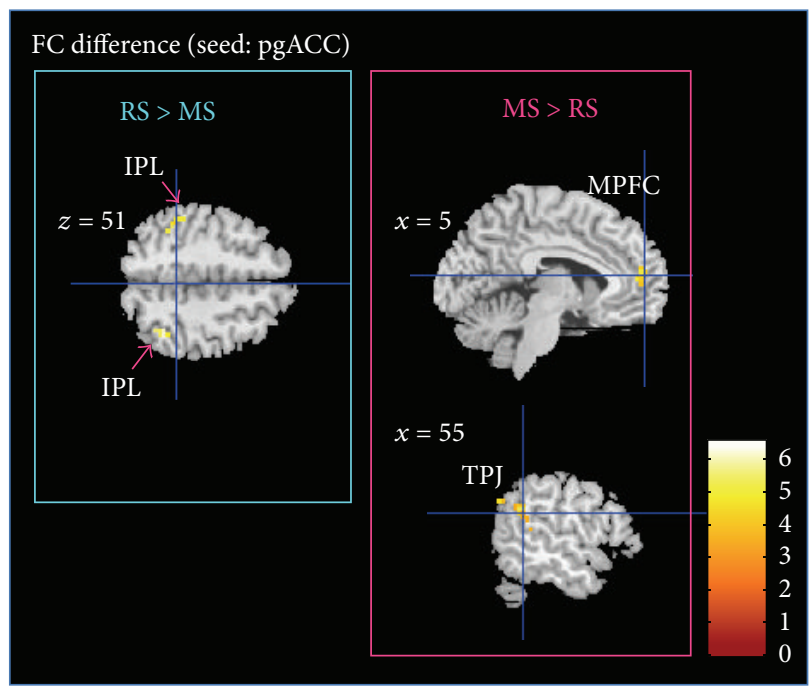

(a)

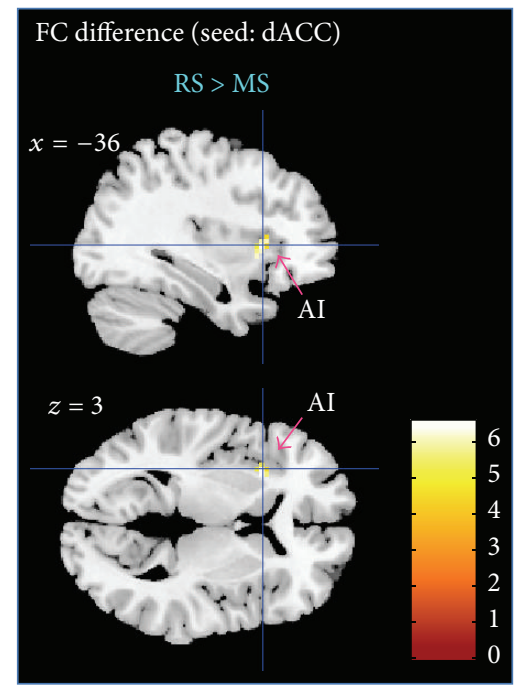

(b)

Figure 2: (a) Paired comparison of seed-based FC maps (seed = pgACC) between rest and meditation at TP2 $(p<0.05$, FWE-corrected). Bar plot represents the $t$-values. White labels indicate the coordinate of each slice in the MNI frame of reference $(x, z)$. (b) Paired comparison of seed-based FC maps (seed = dACC) between rest and meditation at TP2. FC decreases between dorsal ACC and left AI during meditation as compared to rest ( $p<0.05$, FWE-corrected). Bar plot represents the $t$-values. White labels indicate the coordinate of each slice in the MNI frame of reference $(x, z)$.

The dACC showed a decreased FC with the left anterior insula (AI) during MS ( $p<0.05$, FWE-corrected); see Figure 2(b) and Table 3.

The voxel-wise significance level was set at $p<0.001$ with a spatial extent threshold of 16 contiguous voxels, yielding a whole brain threshold of $p<0.05$ corrected for multiple comparisons using AlphaSim algorithm implemented in AFNI (data dimension: $61 \times 73 \times 61$ voxels, Gaussian filter widths: FWHM $x=6.66, \mathrm{FHWM} y=6.88$, and $\mathrm{FWHM} z=$ 6.79). 


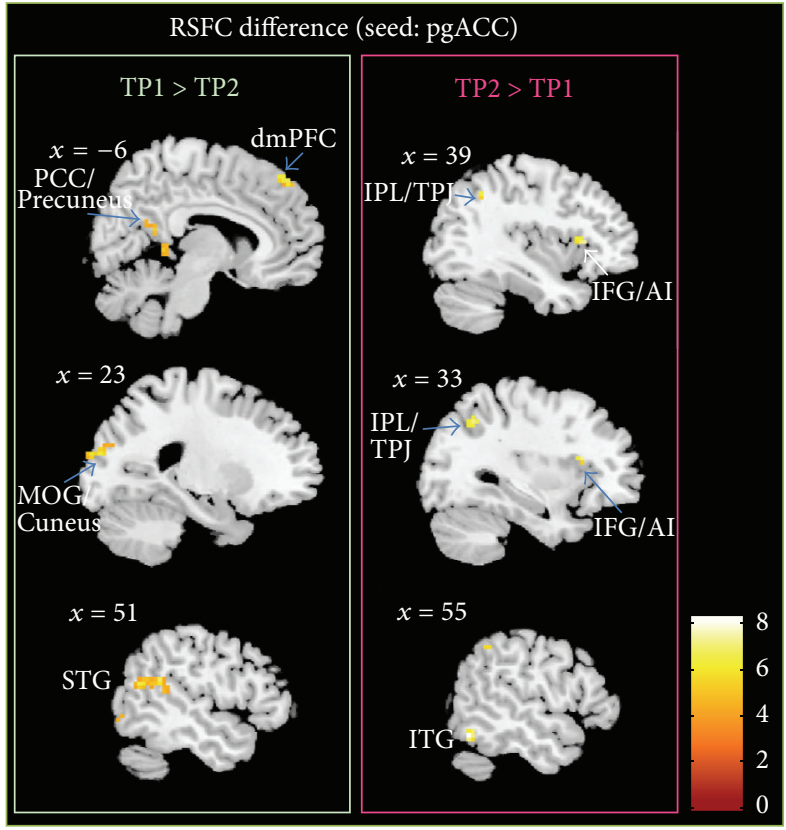

(a)

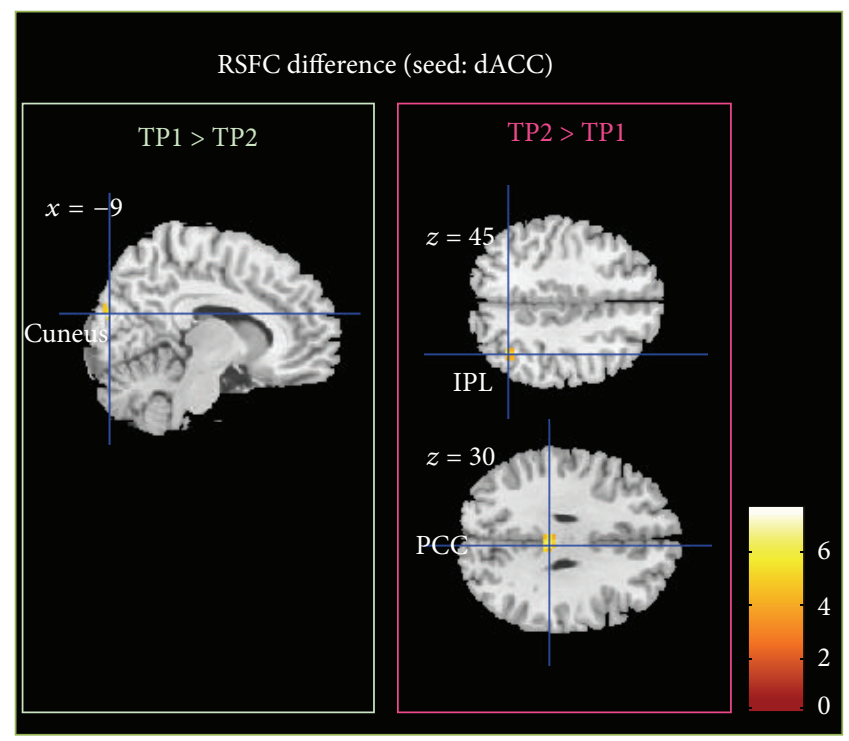

(b)

Figure 3: (a) Longitudinal seed-based RSFC results (seed = pgACC) $(p<0.05$, FWE-corrected). Bar plot represents the $t$-values. White labels indicate the coordinate of each slice in the MNI frame of reference $(x, z)$. (b) Longitudinal seed-based RSFC results (seed $=\mathrm{dACC}$ ) $(p<0.05$, FWE-corrected). Bar plot represents the $t$-values. White labels indicate the coordinate of each slice in the MNI frame of reference $(x, z)$.

3.2.2. Longitudinal Analysis of RSFC Changes due to Meditation. The longitudinal effect of meditation practice (TP1 $>$ TP2) on RSFC was tested separately for pgACC and dACC. For pgACC, a decreased connectivity to the left $\mathrm{PCC} /$ precuneus, the left dorsal medial prefrontal cortex (dmPFC), the right superior temporal gyrus (STG), the left middle occipital gyrus, and left inferior temporal gyrus was observed, while connectivity to the right inferior temporal gyrus, the right inferior frontal gyrus (IFG), and the right TPJ/IPL increased after meditation training $(p<0.05$, FWEcorrected; see Figure 3(a) and Table 4).

In dACC, meditation training entailed a reduction of RSFC to calcarine sulcus and the cuneus but an increased RSFC towards cerebellum, the right inferior parietal lobe (IPL), and the posterior cingulated cortex (PCC) $(p<0.05$, FWE-corrected; see Figure 3(b) and Table 4).

The voxel-wise significance level was set at $p<0.001$ with a spatial extent threshold of 16 contiguous voxels, yielding a whole brain threshold of $p<0.05$ corrected for multiple comparisons using AlphaSim algorithm implemented in AFNI (data dimension: $61 \times 73 \times 61$ voxels, Gaussian filter widths: FWHM $x=6.66$, FHWM $y=6.88$, and FWHMz = 6.79).

\subsection{Group Independent Component Analysis}

3.3.1. Comparison of the DMN Independent Component between $R S$ and MS. To ensure that our DMN comparison between these two conditions was performed in the same low frequency band, power density spectra were obtained. The results revealed that both DMNs were derived from the same

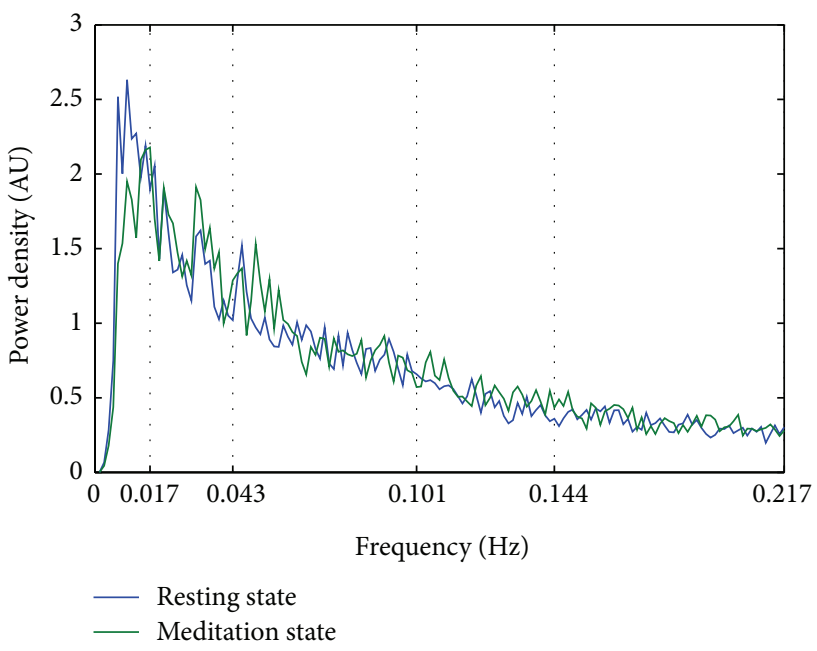

FIgURE 4: Mean power spectral density of the Default Mode Network (DMN) component. The peak power density was observed below $0.04 \mathrm{~Hz}$ for both conditions (resting state and meditation state). AU stands for arbitrary unit.

very low frequency band $(<0.04 \mathrm{~Hz})$. This insured that the comparison between RS and MS were performed in the same “expected" very low frequency domain [60]; see Figure 4.

At TP2, ICA revealed reduced activation during meditation in dACC, sgACC, bilateral insula, superior frontal gyrus, and inferior frontal gyrus (IFG) when compared to rest $(p<0.05$, FWE-corrected). The precuneus and the left 
TABLE 4: Regions showing RSFC differences between time points.

\begin{tabular}{|c|c|c|c|c|c|c|c|c|c|c|}
\hline \multirow{2}{*}{ Analysis } & \multicolumn{5}{|c|}{$\mathrm{TP} 1>\mathrm{TP} 2$} & \multicolumn{5}{|c|}{ TP2 $>$ TP1 } \\
\hline & Region & Side & $K$ & MNI coordinates & $z$-score & Region & Side & $K$ & MNI coordinates & $z$-score \\
\hline ICA & \multicolumn{10}{|c|}{ n.s. $\left(p<0.001, \mathrm{FWE}^{\mathrm{a}} \text {-corrected cluster threshold at } p<0.05\right)^{\mathrm{b}}$} \\
\hline Seed-based FC & \multicolumn{10}{|c|}{$(p<0.001, \text { FWE-corrected cluster threshold at } p<0.05)^{\mathrm{b}}$} \\
\hline \multirow[t]{5}{*}{ (ROI: pgACC) } & PCC/precuneus & $\mathrm{L}$ & 58 & $-12-5115$ & 4.71 & ITG & $\mathrm{R}$ & 16 & $54-63-18$ & 4.23 \\
\hline & $\mathrm{dmPFC}$ & $\mathrm{L}$ & 49 & -124554 & 4.35 & IFG & $\mathrm{R}$ & 17 & 39246 & 3.84 \\
\hline & STG & $\mathrm{R}$ & 53 & $54-6315$ & 4.34 & IPL/TPJ & $\mathrm{R}$ & 18 & $33-6042$ & 3.76 \\
\hline & MOG & $\mathrm{L}$ & 22 & $-24-1026$ & 4.19 & & & & & \\
\hline & ITG & $\mathrm{L}$ & 21 & $-570-24$ & 4.01 & & & & & \\
\hline \multirow[t]{3}{*}{ (ROI: dACC) } & Calcarine/cuneus & $\mathrm{L}$ & 18 & $-9-8421$ & 4.37 & Cerebellum & $\mathrm{L}$ & 22 & $-30-54-30$ & 4.56 \\
\hline & BA19 & $\mathrm{L}$ & 26 & $-27-9021$ & 4.01 & IPL & $\mathrm{R}$ & 16 & $36-6045$ & 4.20 \\
\hline & & & & & & PCC & $\mathrm{R}$ & 19 & $\begin{array}{lll}3 & -30 & 30\end{array}$ & 4.13 \\
\hline
\end{tabular}

ReHo n.s. $(p<0.001 \text {, FWE-corrected cluster threshold at } p<0.05)^{\mathrm{b}}$

Note: dmPFC, dorsal medial prefrontal cortex; IFG, inferior frontal gyrus; IPL, inferior parietal lobe; ITG, inferior temporal gyrus; $K$, cluster size; PCC, post cingulate cortex; STG, superior temporal gyrus; MOG, middle occipital gyrus; and BA: brodmann area.

${ }^{a}$ Family-wise error.

${ }^{\mathrm{b}}$ A combined threshold of $p<0.001$ and a minimum cluster size determined by AlphaSim ${ }^{\mathrm{c}}$ algorithm in AFNI, resulting in corrected threshold of $p<0.05$. An estimate of the spatial correlation across voxels was modeled using the program $3 \mathrm{dFWHM}^{\mathrm{d}}$ in AFNI.

${ }^{c}$ AlphaSim: http://afni.nimh.nih.gov/pub/dist/doc/manual/AlphaSim.pdf.

d 3dFWHM: http://afni.nimh.nih.gov/pub/dist/doc/program_help/3dFWHM.html.

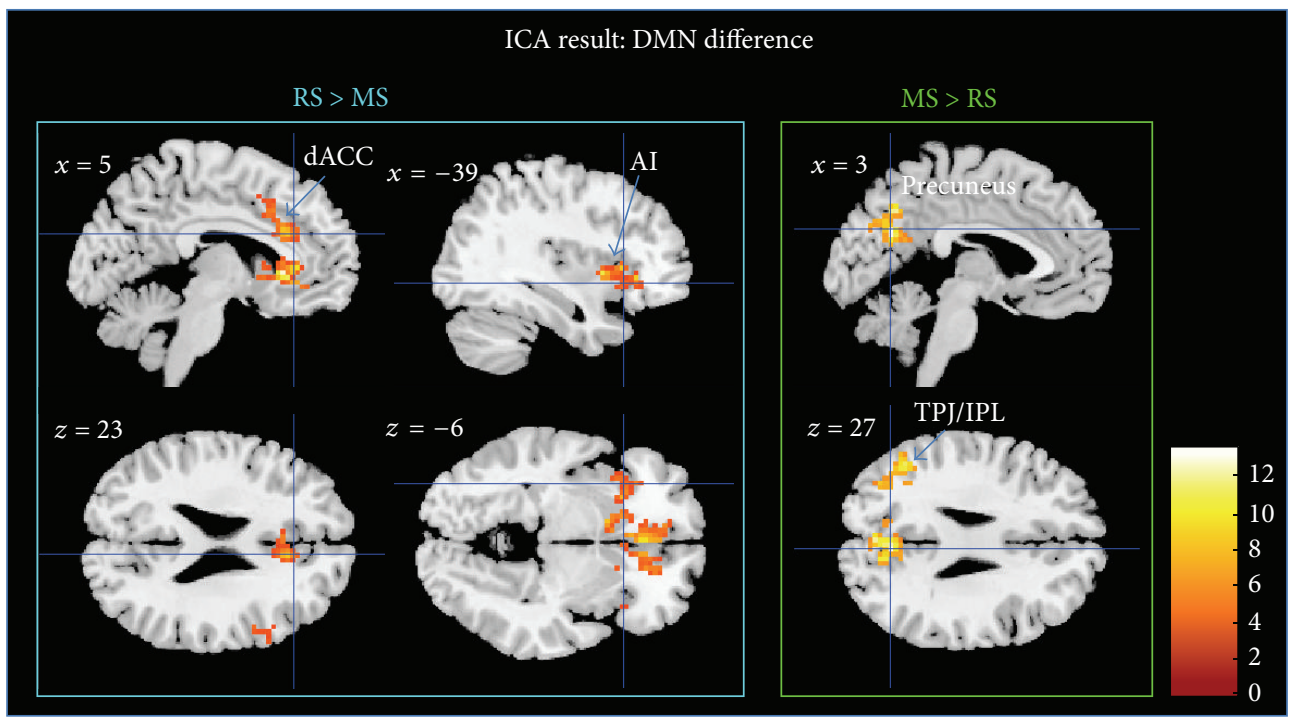

FIGURE 5: ICA results. DMN differences at TP2 for RS $>$ MS and MS $>$ RS ( $p<0.05$, FWE-corrected). Bar plot represents the $t$-values. White labels indicate the coordinate of each slice in the MNI frame of reference $(x, z)$.

temporoparietal junction (TPJ) showed an increased internal consistency in the ICs during MS; see Figure 5 and Table 3.

The voxel-wise significance level was set at $p<0.001$ with a spatial extent threshold of 16 contiguous voxels, yielding a whole brain threshold of $p<0.05$ corrected for multiple comparisons using AlphaSim algorithm implemented in AFNI (data dimension: $61 \times 73 \times 61$ voxels, Gaussian filter widths: FWHM $x=7.39$, FHWM $y=8.18$, and FWHM $z=8.19$ ).

3.3.2. Longitudinal Analysis of RS Changes in the DMN Independent Component due to Meditation. The paired $t$ test at a threshold of $p<0.05$, FWE-corrected, did not reveal longitudinal changes within the DMN independent components. The voxel-wise significance level was set at $p<0.001$ with a spatial extent threshold of 23 contiguous voxels, yielding a whole brain threshold of $p<0.05$ corrected for multiple comparisons using AlphaSim algorithm implemented in AFNI (data dimension: $61 \times 73 \times 61$ voxels, Gaussian filter widths: FWHM $x=7.84$, FHWM $y=8.68$, and FWHM $z=8.53$ ).

\subsection{Regional Homogeneity (ReHo) Analysis}

3.4.1. ReHo Comparison between MS and RS. The comparison of MS and RS (MS > RS) showed an increased ReHo in dACC, left striatum (putamen), MPFC, and TPJ 


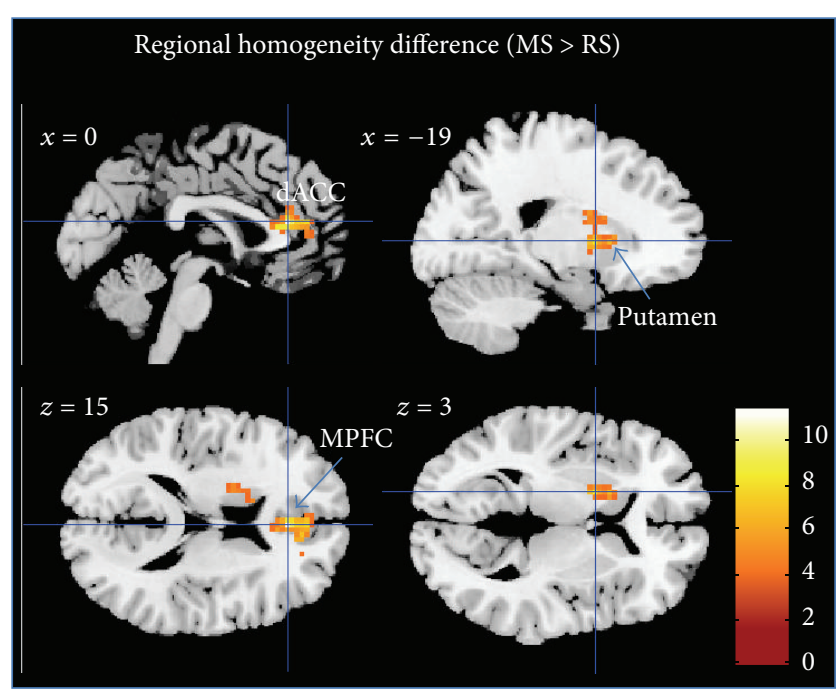

FIGURE 6: Regional homogeneity difference between MS and RS $(p<0.05$, FWE-corrected). Bar plot represents the $t$-values. White labels indicate the coordinate of each slice in the MNI frame of reference $(x, z)$.

(supramarginal gyrus), FWE-corrected at $p<0.05$; see Figure 6 and Table 3.

The voxel-wise significance level was set at $p<0.001$ with a spatial extent threshold of 63 contiguous voxels, yielding a whole brain threshold of $p<0.05$ corrected for multiple comparisons using AlphaSim algorithm implemented in AFNI (data dimension: $61 \times 73 \times 61$ voxels, Gaussian filter widths: FWHM $x=13.25$, FHWM $y=13.98$, and FWHM $z=$ 12.13).

3.4.2. Longitudinal Analysis of ReHo Changes. Longitudinal effects of meditation on the ReHo of RS activity were tested between time points using a paired $t$-test. However, no significant differences between time points were found. The voxel-wise significance level for this analysis was set at $p<$ 0.001 with a spatial extent threshold of 70 contiguous voxels, yielding a whole brain threshold of $p<0.05$ corrected for multiple comparisons using AlphaSim algorithm implemented in AFNI (data dimension: $61 \times 73 \times 61$ voxels, Gaussian filter widths: FWHM $x=14.28$, FHWM $y=14.53$, and FWHM $z=12.48$ ).

\section{Discussion}

4.1. Summary. In the current study, for the first time, we investigated longitudinal effects of mindfulness meditation training on changes in functional connectivity between brain areas. To our knowledge, no previous study has directly compared a meditation condition with a resting condition using sophisticated methods like ICA, ReHo, and FC. During the analyses, we specifically focused on target regions and resting state network playing a role in affective disorders like depression such as the pgACC, the AACC, and the DMN.

We found significant differences in functional connectivity both between states (rest versus meditation) and between time points (before versus after training). The ICA analysis showed differences in the internal consistency in the precuneus and the temporoparietal junction, increased during meditation, while the internal consistency of frontal brain regions decreased. The dACC further revealed reduced connectivity with anterior insula during meditation. As an indication of plastic changes following mindfulness meditation, reduced RSFC between the pgACC and dorsal medical prefrontal cortex was observed after meditation training.

\subsection{Detailed Findings, Previous Work, and Explanations}

4.2.1. Imaging Findings. ICA revealed that, during MS, in comparison to RS, the DMN component had stronger association of TPJ and precuneus, while activity in frontal, cingulate, and insular cortex was less associated with DMN. This may reflect the expected network dissociation as a function of cognitive task during meditation.

4.2.2. Psychometry Findings. Our findings indicate that the short-term practice of mindfulness meditation leads to differences in CES-D score before and after training. Nearly $50 \%$ reduction in depressive symptoms after mindfulness meditation training is consistent with recent meta-analyses reported by $[3,64]$.

Short-term meditation training yielded lowered acute feelings of tension at the time of scanning in our study. Likewise, our results emulate previous reports on the effects of meditation on well-being in anxiety trait and depression self-reports [65].

4.2.3. Implication for Acute Effects and Plasticity Induction during Supporting Therapeutic Efficacy of Meditation. The reported beneficial effects of mindfulness meditation for the treatment of emotional dysregulation in major depression and other affective disorders led us to hypothesize longitudinal changes in the RSFC. Changes in self-reports suggest that meditation training are very well in accordance with enduring changes in brain function as found in the literature and also in our study $[66,67]$. In this sense, the longitudinal observation of RSFC decreases is very well in line with cross-sectional observations of altered functional responses [25] and RS connectivity, especially in the DMN. As one key region mediating depression-related symptoms, pgACC showed a longitudinal reduction in its connectivity with the PCC/precuneus region and conversely increased its connectivity with the right IPL. Such a reduction of intrinsic connectivity between $\mathrm{pgACC}$ and the posterior DMN components in precuneus would indeed reflect the expected directionality of connectivity changes if a hyperconnectivity within DMN in major depressive disorder (MDD), being considered a target of the antidepressant efficacy of meditation training. Sheline and colleagues [20] reported that such hyperconnectivity in the DMN exists for MDD; and, in their study, they highlighted the importance of abnormal hyperconnectivity of a "dorsal nexus." Quite consistently, we also found a reduction of connectivity between pgACC and dmPFC, close to the previously reported dorsal nexus region. That such changes of RSFC after interventions of antidepressant action can 
also be observed in healthy subjects was recently supported by a finding from Scheidegger and colleagues [68], who showed that, similar to our finding, a connectivity decrease of anterior and posterior DMN components, located in pgACC and PCC, was observed after 24 hrs of ketamine injection, mirroring the substances' maximum antidepressant effects in patients. In other words, the neuronal effects observed after 40 days of meditation, which was shown to affect depression-related psychometry, also reduced connectivity in a network that is hyperconnected in MDD, and, given the predominance of pgACC findings, this further suggests some specificity given the overall role of its dysfunction and abnormal connectivity in depression [29].

Longitudinal meditation training effects on functional connectivity are expected to be subserved by acute meditation effects when compared to resting conditions. Similar to the observed longitudinal reduction of RSFC between anterior and posterior DMN components we found an opposite pattern of connectivity changes from rest for pgACC compared to lateral and medial parietal DMN subcomponents. At the same time, the frontal DMN subcomponents seemed to aggregate as indicated by the increased RSFC between pgACC and dmPFC during meditation. Next to within-network connectivities our results showed convergence of state (between conditions) and trait (between time points) effects on the alteration of the functional connectivity between pgACC and right TPJ. Here increased connectivities between these two regions would be in line with functional role of TPJ in reorienting of attention [69]. In the meditation technique applied here, both focused attention and open monitoring aspects were combined. While increased pgACCTPJ connectivity very well fits together with an improvement of open monitoring behavior such as "present moment awareness," it would equally well fit into potential antidepressant efficacy reversing narrowed or biased attention [30], although we did not include a direct measure of such behavioral effects.

When investigating the effects on the attention maintenance network [27], dACC was found to exert a reduced connectivity with its functional counterpart in the AI in MS compared to RS (Figure 2(b)). Previous studies reported insula activity during meditation [12] and anatomical changes in terms of increased gray matter $[70,71]$. The dACC region was however not found to show increased gray matter in either study. This structural distinction of subregions with and without volumetric differences within the cinguloopercular network would be in line with our structural decoupling during meditation. In the literature there are also findings of increases in dACC activity, especially in expert meditators [72] and recent investigations of focused breathing versus mind wandering reported increased activation in both dACC and AI [36]. When interpreting our findings in line with functional involvements of $\mathrm{AACC}$ and AI, next to specifications of the explicit study design, one also has to acknowledge that one region can be part of several networks. This is especially true for AI, which next to its involvement in focused attention, together with dACC, is activated together with MPFC during self-referential conditions and further plays an important role in orchestrating different networks
[73]. In contrast to the observed decoupling of AI and dACC in our seed-based analysis, the finding of reduced correlation with the DMN independent components of these two regions appears within a general observation of reduced IC connectivity in all task-positive regions (Figure 5). Here dACC and AI both showed similar effects of functional decoupling with DMN as an effect of differentiated task behavior during meditation, which however does not imply that they were necessarily any more functionally coupled. The differential effects on AACC and AI are also supported by the local metrics of the ReHo analysis.

Here, the comparison between RS and MS conditions revealed increased ReHo during MS in the MPFC and the $\mathrm{dACC}$ and subcortical regions but not in insula cortices. Since this is the first paper to our knowledge to apply ReHo measures to resting or active states in meditators, we cannot directly relate our observations to other findings. When trying to relate changes of temporal synchrony [62] to crosssectional changes in patient populations, one likewise has to acknowledge that for MPFC both increases and decreases have been reported. While decreases have been for MPFC and ACC in depression [74], but also social anxiety [75] or Alzheimer disease [76], a recent study in bipolar depression rather indicated an increase in MPFC ReHo [74]. If indeed the functional distinction in increased versus decreased ReHo in bipolar versus MDD patients would mirror functional states that also discern rest versus active meditation, this would need to be subject to future investigations targeting the specificity and the physiological meaning of this observation. One important addition to the other connectivity-based findings is however provided by altered ReHo in putamen. This structure has been repeatedly reported in meditation studies $[14,77-80]$ but, distinct from the other main regions frequently reported, was not revealed by our connectivity analyses.

4.3. Strengths and Limitations of the Current Study. Mindfulness meditation and its neural correlates have been investigated in several studies $[36,81]$ by means of block-design fMRI. A limitation of such a design is that meditation is difficult to perform with a short on-and-off period (30$45 \mathrm{sec}$ ), especially for novice meditators. This limitation also results from the GLM itself, in which a longer epoch will decrease the design efficiency. However, the study of Bærentsen et al., 2010, investigated continuous meditation by means of fMRI. They performed both SPM GLM and ICA on continuous meditation and focused on the difference between the meditation onset period and the maintained state of meditation.

One may argue that independent component comparison still would best be performed for components identified across all conditions. In our case, however, similar to Harrison et al., 2008, who used a continuous sad mood induction paradigm compared to RS, we decided to decompose independent components for both conditions separately, also following the method of Calhoun et al., 2008, who used ICASSO to insure ICA stability.

As we did not recruit a control group, our findings cannot be causally attributed solely to mindfulness meditation so 
this will be important improvement for future directions of further investigation.

Moreover, the small sample size, due to discarding of incomplete subjects and imaging artifacts due to motion inside the scanner, limits the generalizability of findings. An increased sample size will increase the statistical power in a future study.

At baseline, the CES-D score of our sample slightly surpassed the clinical cutoff for depression (CES-D > 16), which could be a potential selection bias. Despite a negative diagnosis of depression based on a personal interview, some of the participants may have had a subclinical depression. In this aspect, our sample may still be representative of the general population due to the high prevalence of subclinical depression in general population; however inference to entirely healthy population is limited.

In our present study we did not collect physiological data. Future studies might consider inclusion of certain parameters, such as heart rate, breath rate, and respiration amplitude. High-frequency heart rate variability (HF-HRV) which is a measure of parasympathetic nervous system output that has been associated with enhanced self-regulation [80] would be of particular interest. Since meditation has been shown to increase HF-HRV, this might serve as a biomarker for meditation training-related effects. Recent findings however also suggest a direct relationship especially between HF-HRV and RSFC of, for example, cingulate regions [82], so that functional interpretation of connectivity changes could thus be directly related to autonomous nervous system tone. Further studies on MDD and other affective disorders may help to clarify the actual role of these changes in emotional regulation after short-term meditation, as well as its therapeutic effects.

\section{Conclusion}

We were able to show that the 40-day mindfulness meditation training resulted not only in amelioration of depressionrelated symptoms but also in changes in whole brain networks towards connectivity states usually found when comparing healthy controls to MDD patients in clinical studies. These longitudinal changes were in part mirrored in short-term effects when directly comparing RS and MS. This was especially evident in the involvement of the temporoparietal junction and its connectivity to anterior cingulate cortex, demonstrating cross-network interaction. Within-network consistency was most strongly affected in the DMN where anterior and posterior subcomponents segregated both longitudinally and in direct comparison of MS and RS. In particular effects in dACC which also showed altered local fluctuations during MS and in consequence strongest decoupling from DMN in direct comparison to RS spatially overlap with a network related to attention maintenance [27]; however individual analysis steps revealed variable cluster locations within anatomical boundaries of the midcingulate cortex. These network effects were in part paralleled by observations of altered local fluctuations, which further supported distinct effects in subregions of the salience network, as also supported by reduced connectivity between insula and dACC. Finally we could again identify the putamen as an important subcortical region during meditation, characterized by increased local synchronization of signal variations. Critically, our results may provide insight into the brain circuits that potentially subserve the plastic antidepressant effects of mindfulness meditation training.

\section{Conflict of Interests}

There is no conflict of interests in this paper.

\section{Acknowledgments}

The authors would like to thank the mindfulness meditation training teacher Raúl Balaguer for his kindness for contributing his knowledge to the mindfulness research, Professor Dr. Daniel Pinazo Calatayud for his enthusiastic contribution to initializing the meditation study, Professor Dr. César Ávila for the kind support in fMRI study, Dr. Alfonso BarrósLoscertales for his full support of research design, data analysis, and paper writing, Dr. Martin Walter for his expertise in clinical affective imaging research and paper preparation, and Viola Borchardt for her kind support in preparing the paper. This study is based on work supported by grants to MINECO (PSI2012-33054) and the Spanish National Drug Strategy (4623/2011) to Alfonso Barrós-Loscertales, as well as Spanish State Secretary of Research MINECO (CSO201234066) to Daniel Pinazo and Martin Walter (DFG-SFB779).

\section{References}

[1] R. Chambers, E. Gullone, and N. B. Allen, "Mindful emotion regulation: an integrative review," Clinical Psychology Review, vol. 29, no. 6, pp. 560-572, 2009.

[2] N. A. S. Farb, A. K. Anderson, and Z. V. Segal, "The mindful brain and emotion regulation in mood disorders," Canadian Journal of Psychiatry, vol. 57, no. 2, pp. 70-77, 2012.

[3] S. G. Hofmann, A. T. Sawyer, A. A. Witt, and D. Oh, “The effect of mindfulness-based therapy on anxiety and depression: a meta-analytic review," Journal of Consulting and Clinical Psycho$\log y$, vol. 78, no. 2, pp. 169-183, 2010.

[4] B. K. Hölzel, J. Carmody, M. Vangel et al., "Mindfulness practice leads to increases in regional brain gray matter density," Psychiatry Research: Neuroimaging, vol. 191, no. 1, pp. 36-43, 2011.

[5] Y.-Y. Tang and M. I. Posner, "Special issue on mindfulness neuroscience," Social Cognitive and Affective Neuroscience, vol. 8, no. 1, pp. 1-3, 2013.

[6] D. J. Siegel, "Mindfulness training and neural integration: differentiation of distinct streams of awareness and the cultivation of well-being," Social Cognitive \& Affective Neuroscience, vol. 2, no. 4, pp. 259-263, 2007.

[7] S. Bowen, N. Chawla, S. E. Collins et al., "Mindfulness-based relapse prevention for substance use disorders: a pilot efficacy trial," Substance Abuse, vol. 30, no. 4, pp. 295-305, 2009.

[8] S. Rosenzweig, J. M. Greeson, D. K. Reibel, J. S. Green, S. A. Jasser, and D. Beasley, "Mindfulness-based stress reduction for chronic pain conditions: variation in treatment outcomes and role of home meditation practice," Journal of Psychosomatic Research, vol. 68, no. 1, pp. 29-36, 2010.

[9] E. L. Garland, S. A. Gaylord, C. A. Boettiger, and M. O. Howard, "Mindfulness training modifies cognitive, affective, and physiological mechanisms implicated in alcohol dependence: results 
of a randomized controlled pilot trial," Journal of Psychoactive Drugs, vol. 42, no. 2, pp. 177-192, 2010.

[10] B. K. Hölzel, E. A. Hoge, D. N. Greve et al., "Neural mechanisms of symptom improvements in generalized anxiety disorder following mindfulness training," NeuroImage: Clinical, vol. 2, pp. 448-458, 2013.

[11] J. A. Brewer, P. D. Worhunsky, J. R. Gray, Y.-Y. Tang, J. Weber, and H. Kober, "Meditation experience is associated with differences in default mode network activity and connectivity," Proceedings of the National Academy of Sciences of the United States of America, vol. 108, no. 50, pp. 20254-20259, 2011.

[12] A. Lutz, J. Brefczynski-Lewis, T. Johnstone, and R. J. Davidson, "Regulation of the neural circuitry of emotion by compassion meditation: effects of meditative expertise," PLoS ONE, vol. 3, no. 3, Article ID e1897, 2008.

[13] V. A. Taylor, V. Daneault, J. Grant et al., "Impact of meditation training on the default mode network during a restful state," Social Cognitive and Affective Neuroscience, vol. 8, no. 1, pp. 414, 2013.

[14] J. A. Brefczynski-Lewis, A. Lutz, H. S. Schaefer, D. B. Levinson, and R. J. Davidson, "Neural correlates of attentional expertise in long-term meditation practitioners," Proceedings of the National Academy of Sciences of the United States of America, vol. 104, no. 27, pp. 11483-11488, 2007.

[15] J. H. Jang, W. H. Jung, D.-H. Kang et al., "Increased default mode network connectivity associated with meditation," Neuroscience Letters, vol. 487, no. 3, pp. 358-362, 2011.

[16] M. D. Fox and M. E. Raichle, "Spontaneous fluctuations in brain activity observed with functional magnetic resonance imaging," Nature Reviews Neuroscience, vol. 8, no. 9, pp. 700-711, 2007.

[17] M. D. Greicius, K. Supekar, V. Menon, and R. F. Dougherty, "Resting-state functional connectivity reflects structural connectivity in the default mode network," Cerebral Cortex, vol. 19, no. 1, pp. 72-78, 2009.

[18] M. E. Raichle, A. M. MacLeod, A. Z. Snyder, W. J. Powers, D. A. Gusnard, and G. L. Shulman, "A default mode of brain function," Proceedings of the National Academy of Sciences of the United States of America, vol. 98, no. 2, pp. 676-682, 2001.

[19] R. B. Mars, F.-X. Neubert, M. P. Noonan, J. Sallet, I. Toni, and M. F. S. Rushworth, "On the relationship between the 'default mode network' and the 'social brain,"' Frontiers in Human Neuroscience, vol. 6, article 189, 2012.

[20] Y. I. Sheline, J. L. Price, Z. Yan, and M. A. Mintun, "Resting-state functional MRI in depression unmasks increased connectivity between networks via the dorsal nexus," Proceedings of the National Academy of Sciences of the United States of America, vol. 107, no. 24, pp. 11020-11025, 2010.

[21] D. A. Gusnard, E. Akbudak, G. L. Shulman, and M. E. Raichle, "Medial prefrontal cortex and self-referential mental activity: relation to a default mode of brain function," Proceedings of the National Academy of Sciences of the United States of America, vol. 98, no. 7, pp. 4259-4264, 2001.

[22] K. N. Ochsner and J. J. Gross, "The cognitive control of emotion," Trends in Cognitive Sciences, vol. 9, no. 5, pp. 242-249, 2005.

[23] C. Lemogne, G. le Bastard, H. Mayberg et al., "In search of the depressive self: extended medial prefrontal network during selfreferential processing in major depression," Social Cognitive and Affective Neuroscience, vol. 4, no. 3, pp. 305-312, 2009.

[24] M. G. Berman, S. Peltier, D. E. Nee, E. Kross, P. J. Deldin, and J. Jonides, "Depression, rumination and the default network,"
Social Cognitive and Affective Neuroscience, vol. 6, no. 5, pp. 548-555, 2011.

[25] M. Walter, A. Henning, S. Grimm et al., "The relationship between aberrant neuronal activation in the pregenual anterior cingulate, altered glutamatergic metabolism, and anhedonia in major depression," Archives of General Psychiatry, vol. 66, no. 5, pp. 478-486, 2009.

[26] G. Bush, P. Luu, and M. I. Posner, "Cognitive and emotional influences in anterior cingulate cortex," Trends in Cognitive Sciences, vol. 4, no. 6, pp. 215-222, 2000.

[27] N. U. F. Dosenbach, D. A. Fair, A. L. Cohen, B. L. Schlaggar, and S. E. Petersen, "A dual-networks architecture of top-down control," Trends in Cognitive Sciences, vol. 12, no. 3, pp. 99-105, 2008.

[28] M.-J. van Tol, N. J. A. van der Wee, O. A. van den Heuvel et al., "Regional brain volume in depression and anxiety disorders," Archives of General Psychiatry, vol. 67, no. 10, pp. 1002-1011, 2010.

[29] D. I. Horn, C. Yu, J. Steiner et al., "Glutamatergic and restingstate functional connectivity correlates of severity in major depression-the role of pregenual anterior cingulate cortex and anterior insula," Frontiers in Systems Neuroscience, vol. 4, article 33, 2010.

[30] S. G. Disner, C. G. Beevers, E. A. P. Haigh, and A. T. Beck, "Neural mechanisms of the cognitive model of depression," Nature Reviews Neuroscience, vol. 12, no. 8, pp. 467-477, 2011.

[31] R. H. Kaiser, J. R. Andrews-Hanna, T. D. Wager, and D. A. Pizzagalli, "Large-scale network dysfunction in major depressive disorder: a meta-analysis of resting-state functional connectivity," JAMA Psychiatry, vol. 72, no. 6, pp. 603-611, 2015.

[32] B. B. Biswal, M. Mennes, X.-N. Zuo et al., "Toward discovery science of human brain function," Proceedings of the National Academy of Sciences of the United States of America, vol. 107, no. 10, pp. 4734-4739, 2010.

[33] D. L. Schacter, D. R. Addis, and R. L. Buckner, "Episodic simulation of future events: concepts, data, and applications," Annals of the New York Academy of Sciences, vol. 1124, no. 1, pp. 39-60, 2008.

[34] F. Schneider, F. Bermpohl, A. Heinzel et al., "The resting brain and our self: self-relatedness modulates resting state neural activity in cortical midline structures," Neuroscience, vol. 157, no. 1, pp. 120-131, 2008.

[35] S. Krüger, D. Seminowicz, K. Goldapple, S. H. Kennedy, and H. S. Mayberg, "State and trait influences on mood regulation in bipolar disorder: blood flow differences with an acute mood challenge," Biological Psychiatry, vol. 54, no. 11, pp. 1274-1283, 2003.

[36] J. Dickenson, E. T. Berkman, J. Arch, and M. D. Lieberman, "Neural correlates of focused attention during a brief mindfulness induction," Social Cognitive and Affective Neuroscience, vol. 8, no. 1, pp. 40-47, 2013.

[37] S. M. Smith, K. L. Miller, G. Salimi-Khorshidi et al., "Network modelling methods for FMRI," NeuroImage, vol. 54, no. 2, pp. 875-891, 2011.

[38] B. Zhang, M. Li, W. Qin et al., "Altered functional connectivity density in major depressive disorder at rest," European Archives of Psychiatry and Clinical Neuroscience, 2015.

[39] J. D. Kendall and M. G. Gibbons, Rank Correlation Methods, Edward Arnold, London, UK, 5th edition, 1990.

[40] Z. Yao, L. Wang, Q. Lu, H. Liu, and G. Teng, "Regional homogeneity in depression and its relationship with separate depressive symptom clusters: a resting-state fMRI study," Journal of Affective Disorders, vol. 115, no. 3, pp. 430-438, 2009. 
[41] Z. Liu, C. Xu, Y. Xu et al., "Decreased regional homogeneity in insula and cerebellum: a resting-state fMRI study in patients with major depression and subjects at high risk for major depression," Psychiatry Research-Neuroimaging, vol. 182, no. 3, pp. 211-215, 2010.

[42] M.-J. van Tol, L. R. Demenescu, N. J. A. van der Wee et al., "Functional magnetic resonance imaging correlates of emotional word encoding and recognition in depression and anxiety disorders," Biological Psychiatry, vol. 71, no. 7, pp. 593-602, 2012.

[43] R. A. Baer, "Mindfulness training as a clinical intervention: a conceptual and empirical review," Clinical Psychology: Science and Practice, vol. 10, no. 2, pp. 125-143, 2006.

[44] J. Kabat-Zinn, Full Catastrophe Living: Using the Wisdom of Your Body and Mind to Face Stress, Pain, and Illness, Rev Upd Edition, Bantam, New York, NY, USA, 1990.

[45] S. C. Hayes, J. B. Luoma, F. W. Bond, A. Masuda, and J. Lillis, "Acceptance and commitment therapy: model, processes and outcomes," Behaviour Research and Therapy, vol. 44, no. 1, pp. $1-25,2006$.

[46] E. Andrade, C. Arce, J. Torrado, J. Garrido, C. De Francisco, and I. Arce, "Factor structure and invariance of the POMS mood state questionnaire in Spanish," Spanish Journal of Psychology, vol. 13, no. 1, pp. 444-452, 2010.

[47] D. M. McNair, M. Lorr, and L. F. Droppleman, Manual for the Profile of Mood States, Educational and Industrial Testing Service, San Diego, Calif, USA, 1971.

[48] L. S. Radloff, “The CES-D scale: a self-report depression scale for research in the general population," Applied Psychological Measurement, vol. 1, no. 3, pp. 385-401, 1977.

[49] C. D. Spielberger, R. L. Gorssuch, P. R. Lushene, P. R. Vagg, and G. A. Jacobs, Manual for the State-Trait Anxiety Inventory, Consulting Psychologists Press, 1983.

[50] S. M. Smith, M. Jenkinson, M. W. Woolrich et al., "Advances in functional and structural MR image analysis and implementation as FSL," NeuroImage, vol. 23, supplement 1, pp. S208-S219, 2004.

[51] C.-G. Yan and Y.-F. Zang, "DPARSF: a MATLAB toolbox for 'pipeline' data analysis of resting-state fMRI," Frontiers in System Neuroscience, vol. 4, article 13, 2010.

[52] B. Biswal, F. Zerrin Yetkin, V. M. Haughton, and J. S. Hyde, "Functional connectivity in the motor cortex of resting human brain using echo-planar MRI," Magnetic Resonance in Medicine, vol. 34, no. 4, pp. 537-541, 1995.

[53] M. J. Lowe, B. J. Mock, and J. A. Sorenson, "Functional connectivity in single and multislice echoplanar imaging using resting-state fluctuations," NeuroImage, vol. 7, no. 2, pp. 119-132, 1998.

[54] K. Murphy, R. M. Birn, D. A. Handwerker, T. B. Jones, and P. A. Bandettini, "The impact of global signal regression on resting state correlations: are anti-correlated networks introduced?" NeuroImage, vol. 44, no. 3, pp. 893-905, 2009.

[55] J. L. Price and W. C. Drevets, "Neurocircuitry of mood disorders," Neuropsychopharmacology, vol. 35, no. 1, pp. 192-216, 2010.

[56] A. Lord, D. Horn, M. Breakspear, and M. Walter, "Changes in community structure of resting state functional connectivity in unipolar depression," PLoS ONE, vol. 7, no. 8, Article ID e41282, 2012.

[57] V. D. Calhoun, T. Adali, G. D. Pearlson, and J. J. Pekar, "A method for making group inferences from functional MRI data using independent component analysis," Human Brain Mapping, vol. 14, no. 3, pp. 140-151, 2001.
[58] S. Chen, T. J. Ross, W. Zhan et al., "Group independent component analysis reveals consistent resting-state networks across multiple sessions," Brain Research, vol. 1239, pp. 141-151, 2008.

[59] V. D. Calhoun, K. A. Kiehl, and G. D. Pearlson, "Modulation of temporally coherent brain networks estimated using ICA at rest and during cognitive tasks," Human Brain Mapping, vol. 29, no. 7, pp. 828-838, 2008.

[60] B. J. Harrison, J. Pujol, H. Ortiz, A. Fornito, C. Pantelis, and M. Yücel, "Modulation of brain resting-state networks by sad mood induction," PLoS ONE, vol. 3, no. 3, Article ID e1794, p. 12, 2008.

[61] A. J. Bell and T. J. Sejnowski, "The 'independent components' of natural scenes are edge filters," Vision Research, vol. 37, no. 23, pp. 3327-3338, 1997.

[62] Y. Zang, T. Jiang, Y. Lu, Y. He, and L. Tian, "Regional homogeneity approach to fMRI data analysis," NeuroImage, vol. 22, no. 1, pp. 394-400, 2004.

[63] P. M. Lewinsohn, J. R. Seeley, R. E. Roberts, and N. B. Allen, "Center for Epidemiologic Studies Depression Scale (CES-D) as a screening instrument for depression among communityresiding older adults," Psychology and Aging, vol. 12, no. 2, pp. 277-287, 1997.

[64] M. De Vibe, A. Bjørndal, E. Tipton, K. T. Hammerstrøm, and K. Kowalski, "Mindfulness based stress reduction (MBSR) for improving health, quality of life, and social functioning in adults," Campbell Systematic Reviews, vol. 8, no. 3, 127 pages, 2012.

[65] S. L. Shapiro, G. E. Schwartz, and G. Bonner, "Effects of mindfulness-based stress reduction on medical and premedical students," Journal of Behavioral Medicine, vol. 21, no. 6, pp. 581-599, 1998.

[66] G. Desbordes, L. T. Negi, T. W. W. Pace, B. A. Wallace, C. L. Raison, and E. L. Schwartz, "Effects of mindful-attention and compassion meditation training on amygdala response to emotional stimuli in an ordinary, non-meditative state," Frontiers in Human Neuroscience, vol. 6, article 292, 2012.

[67] H. A. Slagter, R. J. Davidson, and A. Lutz, "Mental training as a tool in the neuroscientific study of brain and cognitive plasticity," Frontiers in Human Neuroscience, vol. 5, p. 17, 2011.

[68] M. Scheidegger, M. Walter, M. Lehmann et al., "Ketamine decreases resting state functional network connectivity in healthy subjects: implications for antidepressant drug action," PLoS ONE, vol. 7, no. 9, Article ID e44799, 2012.

[69] N. U. F. Dosenbach, D. A. Fair, F. M. Miezin et al., "Distinct brain networks for adaptive and stable task control in humans," Proceedings of the National Academy of Sciences of the United States of America, vol. 104, no. 26, pp. 11073-11078, 2007.

[70] S. W. Lazar, C. E. Kerr, R. H. Wasserman et al., "Meditation experience is associated with increased cortical thickness," NeuroReport, vol. 16, no. 17, pp. 1893-1897, 2005.

[71] B. K. Hölzel, U. Ott, H. Hempel et al., "Differential engagement of anterior cingulate and adjacent medial frontal cortex in adept meditators and non-meditators," Neuroscience Letters, vol. 421, no. 1, pp. 16-21, 2007.

[72] A. Lutz, L. L. Greischar, D. M. Perlman, and R. J. Davidson, "BOLD signal in insula is differentially related to cardiac function during compassion meditation in experts vs. novices," NeuroImage, vol. 47, no. 3, pp. 1038-1046, 2009.

[73] V. Menon and L. Q. Uddin, "Saliency, switching, attention and control: a network model of insula function," Brain Structure \& Function, vol. 214, no. 5-6, pp. 655-667, 2010. 
[74] C.-H. Liu, X. Ma, F. Li et al., "Regional homogeneity within the default mode network in bipolar depression: a resting-state functional magnetic resonance imaging study," PLOS ONE, vol. 7, no. 11, Article ID e48181, 2012.

[75] C. Qiu, W. Liao, J. Ding et al., "Regional homogeneity changes in social anxiety disorder: a resting-state fMRI study," Psychiatry Research. Neuroimaging, vol. 194, no. 1, pp. 47-53, 2011.

[76] Z. Zhang, Y. Liu, T. Jiang et al., "Altered spontaneous activity in Alzheimer's disease and mild cognitive impairment revealed by Regional Homogeneity," NeuroImage, vol. 59, no. 2, pp. 14291440, 2012.

[77] T. W. Kjaer, C. Bertelsen, P. Piccini, D. Brooks, J. Alving, and H. C. Lou, "Increased dopamine tone during meditation-induced change of consciousness," Cognitive Brain Research, vol. 13, no. 2, pp. 255-259, 2002.

[78] S. W. Lazar, G. Bush, R. L. Gollub, G. L. Fricchione, G. Khalsa, and $\mathrm{H}$. Benson, "Functional brain mapping of the relaxation response and meditation," Neuroreport, vol. 11, no. 7, pp. 15811585, 2000.

[79] Y.-Y. Tang, Y. Ma, Y. Fan et al., "Central and autonomic nervous system interaction is altered by short-term meditation," Proceedings of the National Academy of Sciences of the United States of America, vol. 106, no. 22, pp. 8865-8870, 2009.

[80] D. J. Libby, P. D. Worhunsky, C. E. Pilver, and J. A. Brewer, "Meditation-induced changes in high-frequency heart rate variability predict smoking outcomes," Frontiers in Human Neuroscience, vol. 6, article 54, 2012.

[81] K. B. Bærentsen, H. Stødkilde-Jørgensen, B. Sommerlund et al., "An investigation of brain processes supporting meditation," Cognitive Processing, vol. 11, no. 1, pp. 57-84, 2010.

[82] C. Chang, C. D. Metzger, G. H. Glover, J. H. Duyn, H.-J. Heinze, and M. Walter, "Association between heart rate variability and fluctuations in resting-state functional connectivity," NeuroImage, vol. 68, pp. 93-104, 2013. 

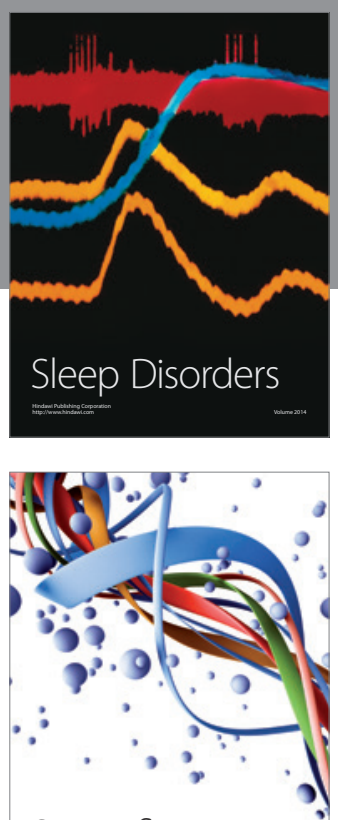

Scientifica
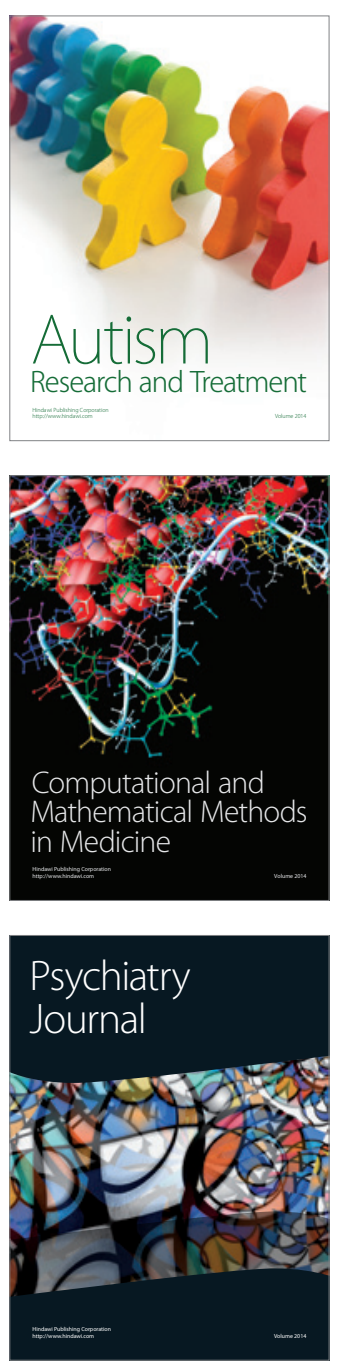
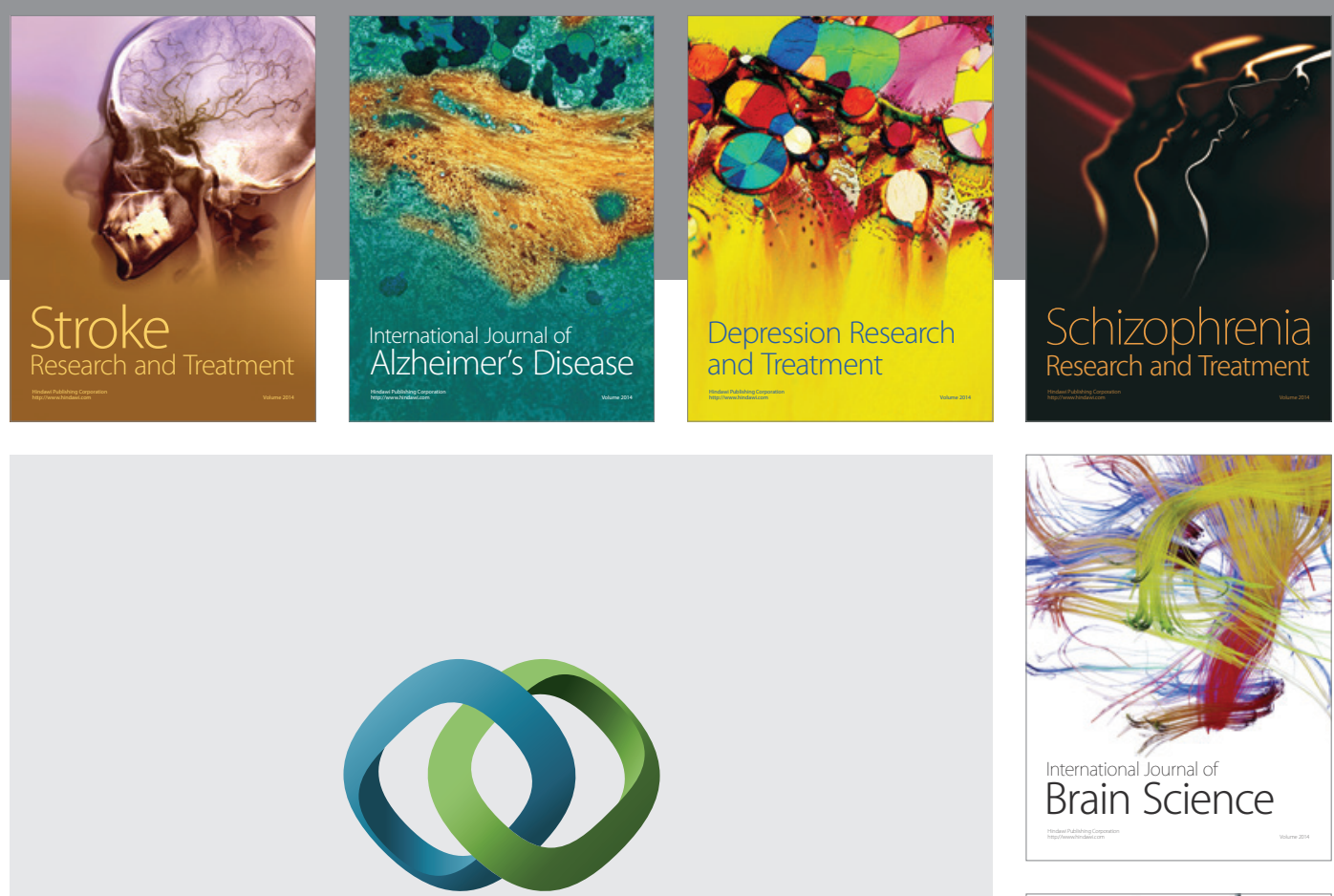

\section{Hindawi}

Submit your manuscripts at

http://www.hindawi.com
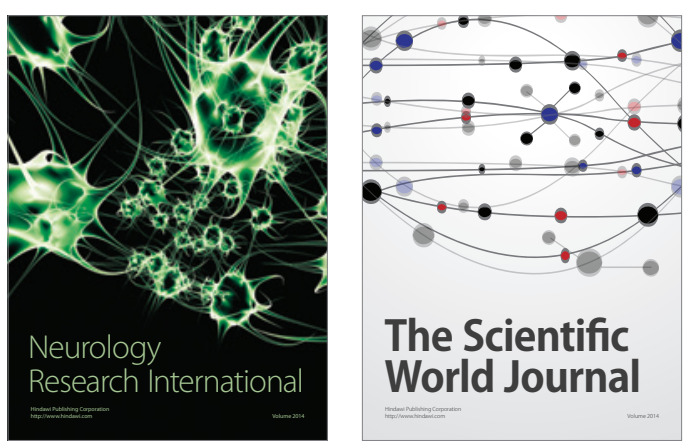

The Scientific World Journal

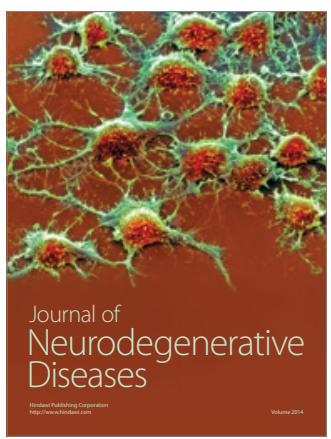

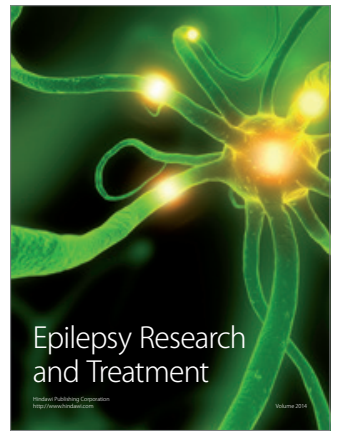

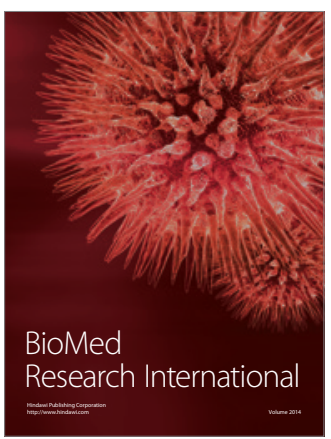

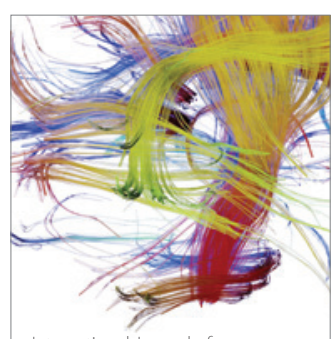

Brain Science

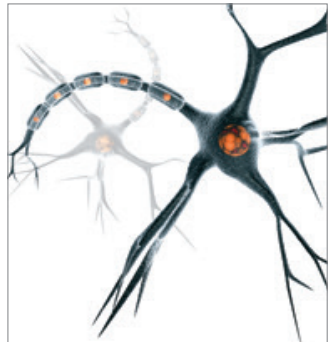

Neural Plasticity
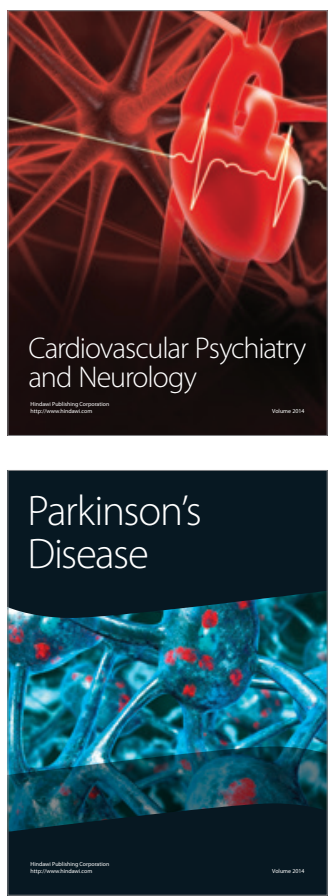